DEUTSCHE AKADEMIE DER WISSENSCHAFTEN ZU BERLIN SCBRIFTEN DER SEKTION FUR ALTERTUMSWISSENSCBAFT

\title{
SENECAS BEWEISFÜHRUNG
}

VoN

WINFRIED TRILLITZSCH

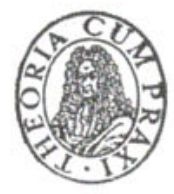

A K A D E M I E - VE R LA G - B E R L I N 
Gutachter dieses Bandes:

Franz Dornseiff $\uparrow$ und Friedrich Zucker

Redaktor der Reihe: Johannes Irmscher

Redaktor dieses Bandes: Ernst Günther Schmidt

Erschienen im Akademie -Verlag GmbH, Berlin W 8, Leipziger Str. 3-4

Copyright 1962 by Akademie -Verlag GmbH, Berlin

Lizenz-Nr. 202 - 100/102/62

Gesamtherstellung: Druckhaus „Maxim Gorki“4, Altenburg

Bestellnummer: 2067/37 - ES 7 M - Preis: DM 24,50 
Meinem Freund und Lehrer

Richard Hahn $†$ 
